\title{
Chemo-immunotherapy - The Role of Monoclonal Antibodies for the Treatment of Chronic Lymphocytic Leukaemia
}

\author{
a report by \\ Michael Hallek \\ German CLL Study Group (GCLLSG), \& Department of Internal Medicine, University of Cologne
}

DOI: $10.17925 / E O H .2006 \cdot 0.2 .73$

\section{Introduction}

The last 15 years have seen a dynamic development of new agents for the treatment of chronic lymphocytic leukaemia (CLL). Fludarabine and a monoclonal antibody, alemtuzumab, have been approved, and additional antibodies (anti-CD20, anti-CD23, rituximab; anti-MHC II) are currently tested in clinical trials. ${ }^{1,2}$ In particular, the combined use of chemotherapy with monoclonal antibodies (commonly called chemo-immunotherapy) has given very promising results in CLL.

\section{Combination Chemotherapy with Purine Analogues}

Fludarabine has been evaluated in a variety of combination regimens. One of the most promising and most thoroughly studied combinations is fludarabine plus cyclophosphamide (FC). ${ }^{3}$

In preliminary, non-comparative trials, the overall response rates did not appear to be better than with fludarabine alone, but the addition of cyclophosphamide appeared to improve the quality of the responses. This combination, with or without mitoxantrone, has achieved response rates of $64 \%$ to $100 \%$, with complete remission (CR) rates of up to $50 \% .^{3}$

In a prospective trial of the German CLL study group (GCLLSG) comparing fludarabine versus FC, results for 375 patients showed superior response rate for the combination. ${ }^{4}$ The FC combination chemotherapy resulted in a significantly higher CR rate $(16 \%)$ and overall response rate $(94 \%)$ compared with fludarabine alone ( $5 \%$ and $83 \% ; \mathrm{p}=0.004$ and 0.001 , respectively).

The FC treatment also resulted in a longer median duration of response (48 vs 20 months; $p=0.001$ ), and a longer event-free survival (49 vs 33 months; $\mathrm{p}=0.001$ ). So far, no difference in the median overall survival could be observed within a median observation period of 22 months. FC caused significantly more thrombocytopenia and neutropenia, but less anaemia than fludarabine. FC did not increase the number of severe infections. ${ }^{4}$

\section{Rituximab - b ased \\ Chemo-immunotherapy}

Rituximab, an anti-CD20 monoclonal antibody, has only recently provoked interest for the treatment of CLL. As a single agent, rituximab is less active than in follicular lymphoma, unless very high doses are used. ${ }^{5,6}$ Somewhat surprisingly, combinations of rituximab with chemotherapy have proven to be very efficacious therapies for CLL.

There is preclinical evidence for synergy between rituximab and fludarabine. ${ }^{7}$ The majority of rituximab combination studies in CLL have focused on combinations with fludarabine or fludarabinebased regimens.

A multicentre phase II study of GCLLSG has evaluated the efficacy and safety of rituximab plus fludarabine in patients with previously treated or untreated CLL. ${ }^{8}$ Of 31 patients treated, 27 (87\%) responded, with 10 patients $(32 \%)$ achieving a complete response.

Byrd and colleagues combined rituximab with fludarabine in either a sequential or concurrent regimen in a randomized study (Cancer and Leukemia Group B (CALGB) 9712 protocol). ${ }^{9}$ Patients $(\mathrm{n}=104)$ with previously untreated CLL received six cycles of fludarabine, with or without rituximab, followed by four once-weekly doses of rituximab.

Overall and complete response rates were higher in the concurrent group $(90 \%$ and $47 \%$ vs $77 \%$ and $28 \%$ ). Similarly, in a large phase II trial conducted at the MD Anderson Cancer Center on 224 patients with previously untreated CLL, rituximab plus FC achieved a response rate of $95 \%$, with $71 \%$ complete responses. ${ }^{10}$

Median overall survival was not reached in patients treated with rituximab plus FC, and was significantly longer than in patients treated with $\mathrm{FC}$ alone in a historical comparison.

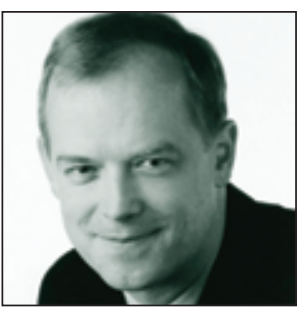

Michael Hallek has been Director of the Department of Internal Medicine at the University of Cologne, Germany, since 2003. The focus of his research is the development of specific, molecular therapies for haematologic malignancies, for which he has received several scientific awards. He is the founder and chairman of the German CLL Study Group, which is currently the largest non-profit multicentre study group on chronic lymphocytic leukaemia worldwide, and chairman of the German Competence Network on Malignant Lymphoma. Professor Hallek studied medicine from 1978 to 1985 in Regensburg, Munich and Paris. He is board certified in internal medicine, haematology and medical oncology. He trained in Munich and Boston, where he also spent two years as research fellow at the Dana Farber Cancer Institute and Harvard Medical School (1990 to 1992). 
Table I: Summary of the Therapeutic Progress as Determined by Recent Clinical Trials on Patients with CLL

\begin{tabular}{llllll} 
& Drugs & $\boldsymbol{n}$ & $\boldsymbol{C R}$ & OR & Survival \\
\hline Rai et al. & CLB & 181 & $4 \%$ & $37 \%$ & 56 \\
& $\mathrm{~F}$ & 170 & $20 \%$ & $63 \%$ & 66 \\
\hline Eichhorst et al. ${ }^{4}$ & $\mathrm{~F}$ & 164 & $18 \%$ & $84 \%$ & $?$ \\
& $\mathrm{FC}$ & 164 & $35 \%$ & $95 \%$ & $?$ \\
\hline Keating, JCO 2005'0 & $\mathrm{FC}+\mathrm{R}$ & 224 & $73 \% *$ & $95 \%$ & $?$ \\
\hline
\end{tabular}

$F=$ Fludarabine; $C L B=$ Chlorambucil; $F C=$ Fludarabine + Cyclophosphamide; $R=$ Rituximab; $C R=$ complete remission; $O R=$ overall response.

* some molecular remissions as determined by polymerase chain reaction.

Alem tuzumab-based

Chemo-immunotherapy

Alemtuzumab is a recombinant, fully humanised, monoclonal antibody against the CD52 antigen. Monotherapy with alemtuzumab has produced response rates of $33-53 \%$, with a median duration of response ranging from 8.7 to 15.4 months, in patients with advanced CLL who were previously treated with alkylating agents and had failed or relapsed after second-line fludarabine therapy. ${ }^{11-13}$

In addition, alemtuzumab has proven effective even in patients with poor prognostic factors, including high-risk genetic markers such as deletions of chromosome 11 or 17 and p53 mutations. ${ }^{14,15}$ If these results are confirmed in larger, prospective trials, alemtuzumab might be a rational choice for first-line treatment of patients with these poor prognostic factors.
O'Brien and colleagues reported an overall response rate of $53 \%$, comprised of nine out of 23 $(39 \%)$ at a $10 \mathrm{mg}$ dose and 17 of $26(65 \%)$ at a $30 \mathrm{mg}$ dose $(p=0.066) .{ }^{18}$ Residual disease was cleared from the bone marrow in most patients, and 11 (38\%) of the 29 patients with available data achieved a molecular remission. Median time to disease progression had not yet been reached for patients who achieved minimal residual disease (MRD) negativity, compared with 15 months for patients who still had residual disease after alemtuzumab consolidation treatment. ${ }^{18}$ While the GCLLSG trial was halted early because of infectious adverse events, the study by O'Brien et al. had no such issue, perhaps due to a longer time interval between induction therapy and consolidation with alemtuzumab (six months vs three months in the GCLLSG study).

Perhaps the most potent regimen for CLL is the combination of the most effective single chemotherapeutic agent with the most effective monoclonal antibody-fludarabine plus alemtuzumab. The synergistic activity of these two agents was initially suggested by the induction of responses, including one $\mathrm{CR}$, in five of six patients who were refractory to each agent alone. ${ }^{19}$ The combination of fludarabine and alemtuzumab was investigated in a phase II trial enrolling patients with relapsed CLL. ${ }^{20}$ Using a four-weekly dosing protocol, this combination has proven feasible, safe and very effective. Among the 36 patients, the objective response rate (ORR) was 83\% (30/36

\section{Impressive progress has been made in the therapy of CLL by using novel chemo-immunotherapies, which combine monoclonal antibodies with purine analogue-based chemotherapy.}

Alemtuzumab consolidation therapy after fludarabinebased chemotherapy also improved the quality of responses, achieved molecular remissions in a substantial proportion of patients and increased progression-free survival (PFS) compared with patients who had no further treatment. ${ }^{16-18}$ Results of a phase III trial by GCLLSG showed improved PFS with alemtuzumab consolidation therapy compared with the observation arm (no progression vs 24.7 months, $\mathrm{p}=0.036$ ) when calculated from the start of fludarabine-based treatment. ${ }^{16}$ When PFS was calculated from the date of alemtuzumab administration, the same benefit was apparent, with no progression compared with 17.8 months $(p=0.036)$. patients), which included $11 \mathrm{CRs}(30 \%)$ and 19 partial remission (PRs) (53\%); in addition, one patient achieved stable disease. Sixteen of 31 (53\%) evaluated patients achieved MRD negativity in the peripheral blood by three months of follow-up, and resolution of disease was observed in all disease sites, particularly in the blood, bone marrow and spleen. Overall, the fludarabine/alemtuzumab combination therapy was fairly well tolerated.

\section{Conclusion}

In the last decade, impressive progress has been made in the therapy of CLL by using novel chemo- 


\title{
BA Bayer HealthCare Bayer Schering Pharma
}

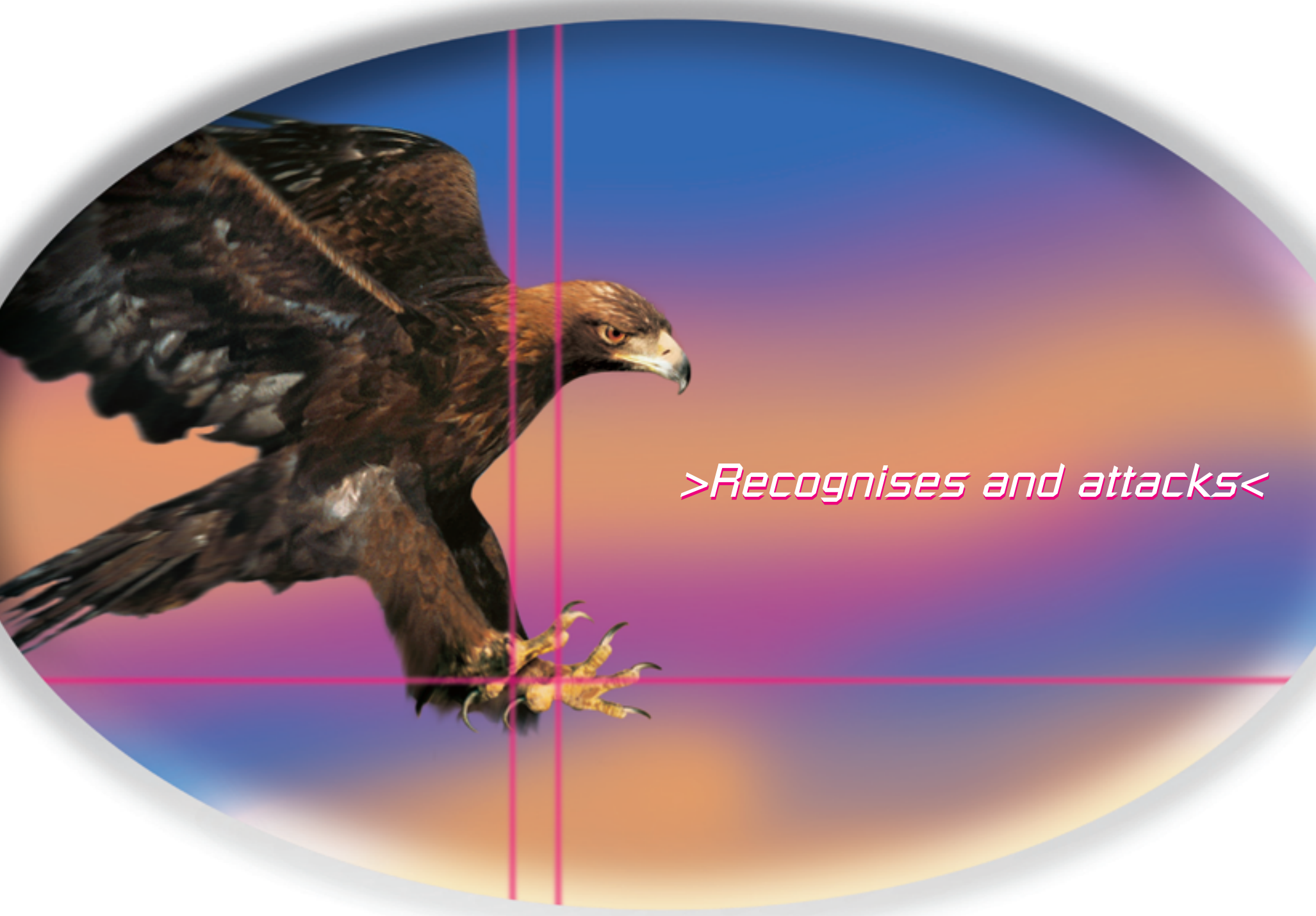

MabCampath ${ }^{\circledR}$ is the first monoclonal antibody for CLL. It can offer substantial improvements in duration of

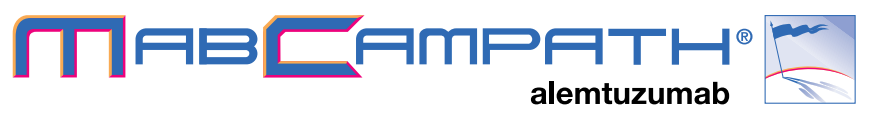
response in all refractory CLL where alkylating agents and fludarabine have failed.

\author{
Target-specific treatment for CLL
}

Prescribing information: Name of the medicinal product MabCampath ${ }^{\oplus} 10$ $\mathrm{mg} / \mathrm{ml}$ concentrate for solution for infusion. MabCampath $30 \mathrm{mg} / \mathrm{ml}$ concentrate for solution for infusion. Qualitative and quantitative $30 \mathrm{mg}$ alemtuzumab. Alemtuzumab is a genetically engineered humanised lgG1 kappa monoclonal antibody specific for a 21-28 kD lymphocyte cell surface glycoprotein (CD52). The antibody is produced in mammalian cell (Chinese Hamster ovary) suspension culture in a nutrient medium. Therapeutic indications: MabCampath ${ }^{\oplus}$ is indicated for the treatment of patients with chronic lymphocytic leukaemia (CLL) who have been treated with alkylating agents and who have failed to achieve a complete or partial response or achieved only a short remission (less than 6 months) following fludarabine phosphate therapy. Contraindications: Hypersensitivity or anaphylactic reactions to alemtuzumab, to murine proteins or to any of the excipients; in patients with active systemic infections; in patients infected with HIV; in patients with active secondary malignancies; pregnancy and breast-feeding. Undesirable effects: More than $80 \%$ patients may be expected to experience adverse reactions; the most commonly reported reactions usually occur during the first week of therapy. The frequencies of the adverse reactions reported below (very common $>10 \%$, common $>1-10 \%$ ) are based on clinical trial data in CLL patients and postmarketing data. Application site: common - injection site reaction. Body as a whole: very common - rigors, fever, fatigue, anorexia; common - back pain, neutropenic fever, chest pain, pain, oedema mouth, asthenia, malaise, influenzalike symptoms, oedema, temperature change sensation. Cardiovascular: very flushing - hapotension, cral and hearing \& special senses: veny common tremgr \& spesthes tremor, hypoaesthesia, dizziness, hyperkinesia, conjunctivitis, paraesthesia,
vertigo. Gastrointestinal, liver \& biliary: very common - vomiting, nausea, diarrhoea; common - abdominal pain, gastrointestinal haemorrhage, stomatitis, mucositis, heaptic function abnormal, constipation, dyspepsia, stomatitis ulcerative, flatulence. Haematological: very common - granulocytopenia; common - pancytopenia, leukopenia, lymphopenia, purpura. Metabolic \& nutritional: common - hyponatraemia, dehydration, weight decrease, hypocalcaemia, thirst. Musculo-skeletal: common - skeletal pain, arthralgia, myalgia. Psychiatric: common - confusion, anxiety, somnolence, depression, insomnia. Resistance mechanism: very common - sepsis, Herpes simplex; common - cytomegalovirus infection, Pneumocystis carinii infection, moniliasis, Herpes zoster, infection, infection fungal, abscess. Respiratory: very common pneumonia, dyspnoea; common - pneumonitis, bronchospasm, sinusitis, Skin \& appendages: very common - urticaria, rash, pruritus, sweating increased Skin \& appendages: very common - urticaria, rash, pruritus, sweating increased common - rash erythematous, bullous eruption. Urinary: common - urinary tract infection. Special warnings and special precautions for use: Acute adverse reactions, which may occur during initial dose escalation due to the release of cytokines, include hypotension, rigors, fever, shortness of breath, chills an rashes. If these events are moderate to severe, then dosing should continue at the same level prior to each dose escalation, with appropriate premedication, until each dose is well tolerated. If therapy is withheld for more than 7 days MabCampath ${ }^{\oplus}$ should be reinstituted with gradual dose escalation. Transient hypotension has occurred in patients receiving MabCampath. Caution should be used in treating patients with ischaemic heart disease, angha and/or in patients recelving anthypets premcenter Mascampated promedication is hydrocortisone 100-200 mg or which maed proce orat anthistamine, 0.9 . diphenhydramine $50 \mathrm{mg}$, and an analgesic, paracetamol $500 \mathrm{mg}$, may be given. In the event that acute infusion reactions persist, the infusion time may be extended up to 8 hours from the time of reconstitution of MabCampath in solution for infusion. Protound lymphocyte depletion, an expected pharmacological effect of MabCampath ${ }^{\circledast}$. inevitably occurs and may be prolonged. CD4 and CD8 T-cell counts begin to rise from weeks 8-12 during treatment and continue to recover for several months following the discontinuation of treatment. The median time to reach a level of 200 cells/ul is 2 months following last infusion with MabCampath ${ }^{\natural}$ but may take 6 months or longer to approximate pretreatment levels. This may predispose patients to opportunistic infections. It is highly recommended that anti-infective prophylaxis (e.g. trimethoprim/sulfamethoxazole 1 tablet twice daily, 3 times weekly, or other prophylaxis against Pneumocystis carinili pneumonia (PCP) and an effective oral anti-herpes agent, such as famciclovir, $250 \mathrm{mg}$ twice daily) should be initiated while on therapy and continued following the completion of treatment with MabCampath ${ }^{\circledast}$ until the CD4+ count has recovered to $200 \mathrm{cells} / \mathrm{\mu l}$ or greater. If $\mathrm{CD} 4$ counts are not obtainable, then patients should remain on antiinfective prophylaxis for 4 months. Patients may have allergic or hypersensitivity reactions to MabCampath ${ }^{\oplus}$ and to murine or chimeric monoclonal antibodies. Males and females of childbearing potential should use effective contraceptive measures during treatment and for 6 months following MabCampath ${ }^{\circledR}$ therapy. No studies have been conducted which specifically address the effect of age on MabCampath disposition and toxicity. In general, older patients (over 65 years of age) tolerate cytotoxic therapy less well than younger individuals. Since CLL occurs commonly in this older age group, these patients should be monitored

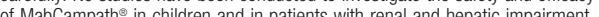
Date of revision of the text: Jnury 2005 Ploase notel For current prescribing informatio prescribing information refer to the package insert and/or contact your local 
immunotherapies, that combine monoclonal antibodies with purine analogue-based chemotherapy. This approach has led to an increased response rate and prolonged treatment-free survival, from complete remissions about $4 \%$ with chlorambucil to up to $70 \%$ with the novel chemoimmunotherapies (see Table 1). So far, it has not been formally proven by prospective, randomised trials that this approach prolongs overall survival of CLL patients. The current protocols such as the CLL8 protocol of the GCLLSG, which compares the $\mathrm{FC}$ versus $\mathrm{FC}+\mathrm{R}$ combination, will contribute to further define the future value of chemoimmunotherapy for CLL.

\section{References}

1. Nagy ZA, Hubner B, Lohning $C$, et al., "Fully human, HLA-DR-specific monoclonal antibodies efficiently induce programmed death of malignant lymphoid cells", Nat Med (2002);8(8): pp. 801-807.

2. Hallek, M, "Chemoimmunotherapy - towards real progress in the treatment of chronic lymphocytic leukemia", Nat Clin Pract Oncol (2005);2(7): pp. 338-339.

3. Hallek $M$, Eichhorst B F, "Chemotherapy combination treatment regimens with fludarabine in chronic lymphocytic leukemia", Hematol J (2004);5 Suppl 1: pp. S20-S30.

4. Eichhorst BF, Busch R, Hopfinger G, et al., "Fludarabine plus cyclophosphamide versus fludarabine alone in first-line therapy of younger patients with chronic lymphocytic leukemia”, Blood (2006);107(3): pp. 885-891.

5. Huhn D, von Schilling C, Wilhelm M, et al., "Rituximab therapy of patients with B-cell chronic lymphocytic leukemia", Blood (2001);98(5): pp. 1326-1331.

6. O'Brien SM, Kantarjian H, Thomas DA, et al., "Rituximab dose-escalation trial in chronic lymphocytic leukemia", J Clin Oncol (2001);19(8): pp. 2165-2170.

7. Di Gaetano N, Xiao Y, Erba E, et al., "Synergism between fludarabine and rituximab revealed in a follicular lymphoma cell line resistant to the cytotoxic activity of either drug alone", Br J Haematol (2001);114(4): pp. 800-809.

8. Schulz H, Klein SK, Rehwald U, et al., "Phase 2 study of a combined immunochemotherapy using rituximab and fludarabine in patients with chronic lymphocytic leukemia", Blood (2002)1;100(9): pp. 3115-3120.

9. Byrd JC, Peterson BL, Morrison VA, et al., "Randomized phase 2 study of fludarabine with concurrent versus sequential treatment with rituximab in symptomatic, untreated patients with B-cell chronic lymphocytic leukemia: results from Cancer and Leukemia Group B 9712 (CALGB 9712)”, Blood (2003);101(1): pp. 6-14.

10. Keating MJ, O'Brien S, Albitar $M$, et al., "Early results of a chemoimmunotherapy regimen of fludarabine, cyclophosphamide, and rituximab as initial therapy for chronic lymphocytic leukemia”, J Clin Oncol (2005);23(18): pp. 4079-4088.

11. Osterborg A, Dyer MJ, Bunjes D, "Phase II multicenter study of human CD52 antibody in previously treated chronic lymphocytic lenkemia. European Study Group of CAMPATH-1H Treatment in Chronic Lymphocytic Lenkemia”, J Clin Oncol (1997);15(4): pp. 1567-1574.

12. Rai KR, Freter CE, Mercier RJ, et al., "Alemtuzumab in previously treated chronic lymphocytic leukemia patients who also had received fludarabine", J Clin Oncol (2002);20(18): pp. 3891-3897.

13. Keating MJ, O'Brien S, Albitar M, et al., "Therapeutic role of alemtuzumab (Campath-1H) in patients who have failed fludarabine: results of a large international study”, Blood (2002);99(10): pp. 3554-3561.

14. Stilgenbauer S, Dohner H, "Campath-1H-induced complete remission of chronic lymphocytic leukemia despite 553 gene mutation and resistance to chemotherapy", N Engl J Med (2002);347(6): pp. 452-453.

15. Lozanski G, Heerema NA, Flinn IW, et al., "Alemtuzumab is an effective therapy for chronic lymphocytic leukemia with p53 mutations and deletions”, Blood (2004);103(9): pp. 3278-3281.

16. Wendtner CM, Ritgen M, Schweighofer CD, et al., "Consolidation with alemtuzumab in patients with chronic lymphocytic leukemia (CLL) in first remission - experience on safety and efficacy within a randomized multicenter phase III trial of the German CLL Study Group (GCLLSG)", emia (2004);18(6): pp. 1093-1101.

17. Montillo M, Cafro AM, Tedeschi A, et al., "Safety and efficacy of subcutaneous Campath-1H for treating residual disease in patients with chronic lymphocytic leukemia responding to fludarabine”, Haematologica (2002);87(7): pp. 695-700.

18. O'Brien SM, Kantarjian HM, Thomas DA, "Alemtuzumab as treatment for residual disease after chemotherapy in patients with chronic lymphocytic leukemia”, Cancer (2003);98(12): pp. 2657-2663.

19.Kennedy B, Rawstron $A$, Carter $C$, et al., "Campath-1H and fludarabine in combination are highly active in refractory chronic lymphocytic leukemia”, Blood (2002);99(6): pp. 2245-2247.

20.Elter T, Borchmann P, Schulz H, et al., "Fludarabine in combination with alemtuzumab is effective and feasible in patients with relapsed or refractory B-cell chronic lymphocytic lenkemia: results of a phase II trial", J Clin Oncol (2005);23(28): pp. 7024-7031.

21.Rai KR, Peterson BL, Appelbaum FR, et al., "Fludarabine compared with chlorambucil as primary therapy for chronic lymphocytic leukemia”, N Engl J Med (2000)4;343(24): pp. 1750-1757. 